\title{
FY90 Milestone Report for the CIT Project: Localizability of Electron-Cyclotron Heating Power
}

\author{
Gary R. Smith \\ Lawrence Livermore National Laboratory \\ University of California \\ Livermore, CA 94550 \\ Subcontract Purchase Order No. S-03119-G with Princeton University \\ 25 October 1990 \\ Estimates of the localizability of electron-cyclotron heating power are made for the \\ Compact Ignition Tokamak. A particular heating scenario is examined, namely, the \\ fundamental $\mathrm{O}$-mode, injected nearly perpendicular to the toroidal magnetic field. The \\ absorption depth due to finite $T_{e}$ is very small, about $1 \mathrm{~cm}$, near the $q=2$ surface. \\ Absorption is even better localized near $q=1$. Several issues that might lead to degraded \\ localizability are reviewed. Use of an intense, pulsed microwave source is the only issue \\ with a possibly significant impact.
}




\section{Introduction}

One of the well-known advantages of electron-cyclotron power for plasma heating is the opportunity to localize the power deposition in a small region of the plasma. In this report we estimate the extent to which electron-cyclotron-heating $(\mathrm{ECH})$ power is localizable in the Compact Ignition Tokamak (CIT).

Two important purposes of localized ECH are tearing-mode and sawtooth suppression. For the former the heating must be localized near the $q=2$ surface, while the latter requires localization near $q=1$. Localization is generally better near $q=1$ than near $q=2$ because higher density and temperature near $q=1$ make the electron-cyclotron absorption stronger. Anticipating the result that localizability is very good at $q=2$ and therefore extremely good at $q=1$, we restrict ourselves to the treatment of the region near $q=2$ in this report.

\section{Intrinsic Localizability of ECH near $q=2$}

We first consider the degree of localization achievable for a microwave beam of negligible angular divergence and spatial width in a quiescent plasma. We make our estimates based on the theoretical results reviewed by Bornatici, et al. in Ref. 1. When the wave damping is weak in the sense that the wavevector is nearly real ( $\operatorname{Im} k \ll \operatorname{Re} k$ ), the spatial power absorption rate is given in Ref. 1 for the ordinary mode near the fundamental resonance by

$$
\begin{aligned}
\alpha & =2 \operatorname{Im} k \cdot S /|S|=2 \operatorname{Im} k \\
& =\frac{1}{2} \operatorname{Re} N_{\perp}\left(\frac{\omega_{p}}{\Omega}\right)^{2} \frac{(\Omega / c)\left[-\operatorname{Im} F_{7 / 2}\left(z_{1}\right)\right]}{\left|1+\frac{1}{2}\left(\omega_{p} / \Omega\right)^{2} \operatorname{Re} F_{7 / 2}\left(z_{1}\right)\right|} .
\end{aligned}
$$

Here, $N_{\perp}$ denotes the perpendicular component of the refractive index. The function $F_{7 / 2}\left(z_{1}\right)$ is shown in Fig. 2 on p. 1183 of Ref. 1, and the distance from the position of exact cyclotron resonance $\Omega=\omega$ is given by the dimensionless variable

$$
z_{1} \equiv \frac{m_{e} c^{2}}{T_{e}} \frac{\omega-\Omega}{\omega} .
$$

The function $-\operatorname{Im} F_{7 / 2}$ has a width of about 5 in $z_{1}$ between the 1/e points, which allows us to estimate the depth of the absorptivity $\alpha$. By itself however, this function determines the depth of the power-deposition profile only in the limit of low opacity.

In terms of $\delta \equiv\left(\omega_{p} / \Omega\right)^{2}$, the opacity is given by

$$
\tau=\pi^{2} \frac{T_{e}}{m_{e} c^{2}} \frac{L_{B}}{\lambda_{0}} \delta(1-\delta)^{1 / 2}\left(1+0.05 \delta^{2}\right)
$$

where $\lambda_{0}=2 \pi c / \omega$ and $L_{B} \equiv B /|d B / d R|$. If $\tau \gg 1$, then the depth of the powerdeposition profile is much less than the depth of the absorptivity $\alpha$.

To estimate the opacity for CIT near the $q=2$ surface, we need values for the quantities appearing in Eq. (3). We take $T_{e}=2 \mathrm{keV}$. From $n_{e}=2 \times 10^{20} \mathrm{~m}^{-3}$ and 
$B=7.5 \mathrm{~T}$, we calculate $\delta=0.37$. The frequency and vacuum wavelength corresponding to cyclotron resonance at $B=7.5 \mathrm{~T}$ are $\omega / 2 \pi=210 \mathrm{GHz}$ and $\lambda_{0}=1.4 \times 10^{-3} \mathrm{~m}$. The beginning-of-flat-top equilibrium calculated in September 1990 by R. Pillsbury has $L_{B}=4.1 \mathrm{~m}$ near $q=2$ along the equatorial plane. Then from Eq. (3) we find $\tau=33$.

We can now estimate the depths $\ell_{\alpha}$ of the absorptivity and $\ell$ of the power-deposition profile. From Eq. (2), the shape of $-\operatorname{Im} F_{7 / 2}$, and $T_{e}=2 \mathrm{keV}$, the absorptivity $\alpha$ is significant over a range of $0.99<\Omega / \omega<1.01$. The corresponding distance is $\ell_{\alpha}=8 \mathrm{~cm}$. For $\tau=33$ we expect a much smaller depth $\ell \approx 1 \mathrm{~cm}$ of the deposition profile.

\section{Effects of Scattering by Drift-Wave Density Fluctuations}

Another report gives a detailed description of our work on the scattering of ECH power by density fluctuations. ${ }^{2}$ Evaluation of the scattering for CIT is included there for turbulence consistent with mixing-length estimates and with observations in a few tokamaks. The expected effect of drift-wave density fluctuations is broadening of the angular divergence of the ECH beam by' about $2^{\circ}$ between the plasma edge and the $q=2$ surface. The scattering effects therefore appear negligible.

\section{Other Effects on Depth of Power-Deposition Profile}

Finite angular divergence or spatial width of the microwave beam could increase the depth of the power-deposition profile. These effects can be made negligible by straightforward design of a standard, circular Gaussian beam with the waist inside the plasma. We emphasize this point by giving here an illustrative design for the final portion of the beam. To direct and focus the beam we assume a final mirror of reasonable size (diameter $=0.4 \mathrm{~m})$ located well away from the plasma $(8 \mathrm{~m}$ from the desired waist position). The variation of the beam width along the beam (direction $z$ ) is described by

$$
w(z)=w_{0}\left[1+\left(z / z_{R}\right)^{2}\right]^{1 / 2},
$$

where $z_{R} \equiv \pi w_{0}^{2} / \lambda_{0}$ is the Rayleigh length. We solve Eq. (4) for $w_{0}$ and substitute values $z=8 \mathrm{~m}, w(z)=0.1 \mathrm{~m}$, and $\lambda_{0}=1.4 \times 10^{-3} \mathrm{~m}$. The result is a radius for the beam waist of $w_{0}=3.9 \mathrm{~cm}$. The Rayleigh length $z_{R}=3.4 \mathrm{~m}$. This Gaussian beam obviously has a negligible width $2 w_{0} \approx 8 \mathrm{~cm}$ compared to the plasma size. Furthermore, the waist can be placed in the region of power deposition $(\Omega=\omega)$, which achieves a flat wavefront (no angular divergence).

The finite curvatures of the cyclotron-resonance and $q=2$ surfaces could increase the power-deposition depth beyond the value $\ell=1 \mathrm{~cm}$ estimated in Sec. II. We have measured the curvatures for the equilibrium mentioned in Sec. II and evaluated the broadening for the beam size given in the previous paragraph. The result is an increase in power-deposition depth $\ell$ that is much less than $1 \mathrm{~cm}$.

If an intense, pulsed (free-electron laser) source is used for the microwave beam in CIT, then the absorption process is described by nonlinear instead of linear theory. ${ }^{3}$. The nonlinear theory has been used to make preliminary estimates for CIT near the $q=2$ 
surface. We find that the strongly nonlinear regime applies, so the power-deposition depth increases and the opacity is reduced significantly. Further analysis of the nonlinear effects is left for future work.

\section{Conclusions}

The intrinsic localizability of an idealized beam absorbed near $q=2$ in a quiescent CIT plasma is about $1 \mathrm{~cm}$, which is much smaller than the plasma minor radius and even smaller than the radial widths of magnetic islands. Localizability is even better near the $q=1$ surface. The following effects have been examined and found to degrade the intrinsic localizability by negligible amounts: scattering by drift-wave density fluctuations, finite beam width and divergence, and curvature of the cyclotron-resonance and $q=2$ surfaces. If an FEL source were used to generate the microwave beam, possibly significant nonlinear effects would arise that affect localizability, but a detailed study of these effects is deferred to a future report. 


\section{References}

${ }^{1}$ M. Bornatici, R. Cano, O. De Barbieri, and F. Engelmann, Nucl. Fusion 23, 1153 (1983).

${ }^{2}$ G. R. Smith, D. R. Cook, and A. N. Kaufman, Scatt ig of electron-cyclotron heating waves by density fluctuations in tokamaks, in preparatıon.

${ }^{3}$ W. M. Nevins, T. D. Rognlien, and B. I. Cohen, Phys. Rev. Lett. 59, 60 (1987). 

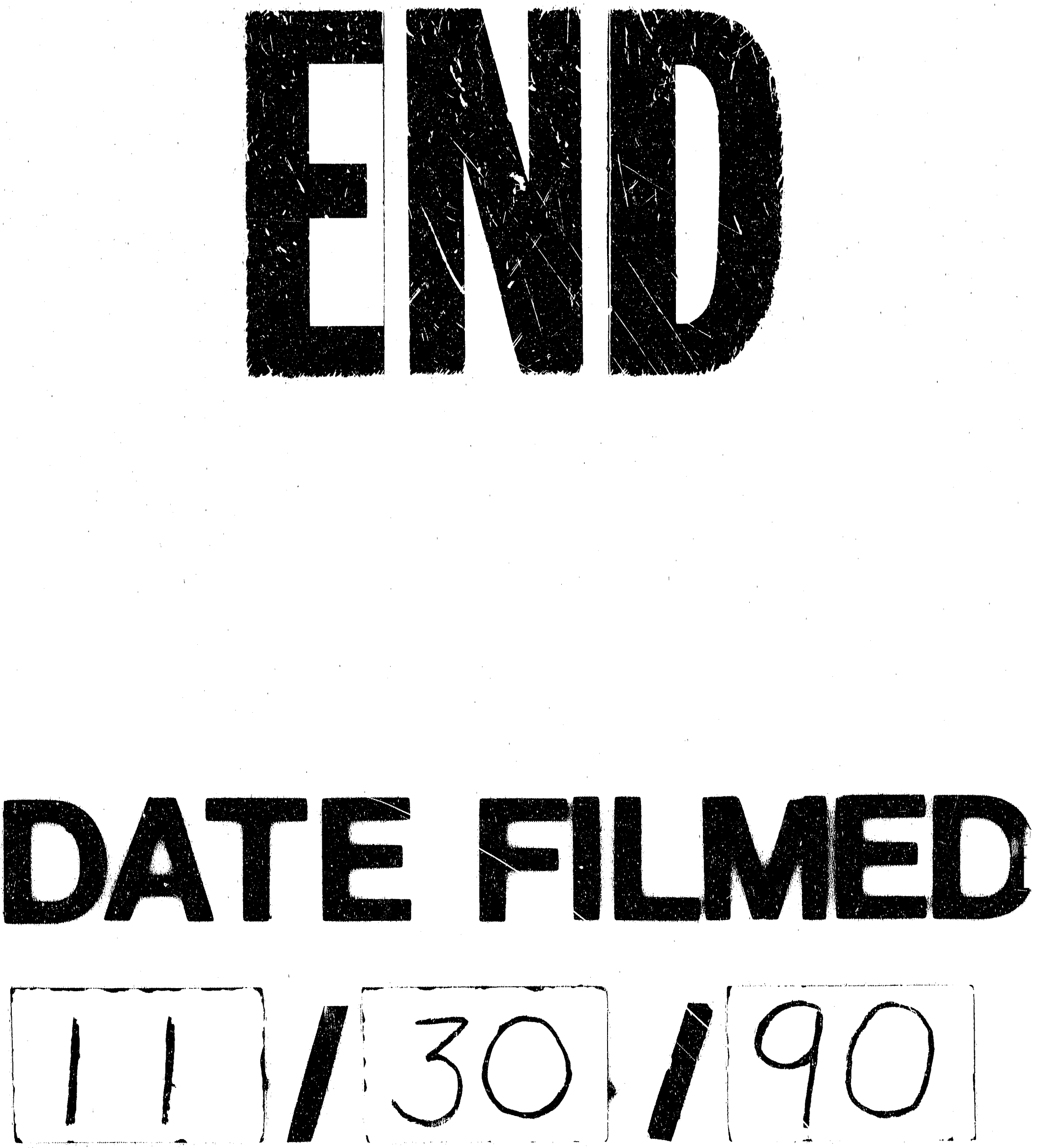
, 